\title{
Combined ICA-LORETA analysis of mismatch negativity
}

\author{
J. Marco-Pallarés, ${ }^{\text {a,b }}$ C. Grau, ${ }^{\text {a,* }}$ and G. Ruffini ${ }^{\mathrm{b}}$ \\ ${ }^{a}$ Neurodynamics Laboratory, Department of Psychiatry and Clinical Psychobiology, University of Barcelona, Passeig de la Vall d'Hebron 171, \\ 08035 Barcelona, Catalonia, Spain \\ ${ }^{\mathrm{b}}$ Starlab, Edif. de l'Observatori Fabra, C. de l'Observatori s/n, Muntanya del Tibidabo, 08035 Barcelona, Spain
}

Received 7 May 2004; revised 1 October 2004; accepted 23 November 2004

Available online 26 January 2005

\begin{abstract}
A major challenge for neuroscience is to map accurately the spatiotemporal patterns of activity of the large neuronal populations that are believed to underlie computing in the human brain. To study a specific example, we selected the mismatch negativity (MMN) brain wave (an event-related potential, ERP) because it gives an electrophysiological index of a "primitive intelligence" capable of detecting changes, even abstract ones, in a regular auditory pattern. ERPs have a temporal resolution of milliseconds but appear to result from mixed neuronal contributions whose spatial location is not fully understood. Thus, it is important to separate these sources in space and time. To tackle this problem, a two-step approach was designed combining the independent component analysis (ICA) and low-resolution tomography (LORETA) algorithms. Here we implement this approach to analyze the subsecond spatiotemporal dynamics of MMN cerebral sources using trial-by-trial experimental data. We show evidence that a cerebral computation mechanism underlies MMN. This mechanism is mediated by the orchestrated activity of several spatially distributed brain sources located in the temporal, frontal, and parietal areas, which activate at distinct time intervals and are grouped in six main statistically independent components.

(C) 2004 Elsevier Inc. All rights reserved.
\end{abstract}

Keywords: Independent component analysis (ICA); Low-resolution tomography (LORETA); Mismatch negativity (MMN)

\section{Introduction}

Mismatch negativity (MMN) is an involuntary auditory eventrelated potential (ERP), which peaks at 100-200 ms when there is a violation (deviant tone) of a regular pattern (standard tone sequence) (Näätänen et al., 1978). The MMN mechanism appears to correspond to a "primitive intelligence," as the wave produced with the violation of regularities - even those of an abstract

\footnotetext{
* Corresponding author. Fax: +34 934034424.

E-mail address: carlesgrau@ub.edu (C. Grau).

URL: http://starlab.es.
}

Available online on ScienceDirect (www.sciencedirect.com). nature - in the auditory stream pattern (Näätänen et al., 2001). Moreover, the deviant information provided by the change needs to be compared with a memory trace of the regularity previously stored in the brain. Thus, MMN provides a promising in vivo simplified model for studying abstract brain processing and related memory mechanisms.

Several cerebral MMN sources have been described in the literature, with global right hemisphere dominance. Studies performed with EEG (Alain et al., 1998), MEG (Rosburg et al., 2004), functional magnetic resonance (fMRI) (Jääskeläinen et al., 2004; Kircher et al., 2004), and positron emission tomography (PET) (Müller et al., 2002) have shown that the main generators of MMN are located in supratemporal cortex. Other contributions to MMN have been described in frontal cortex using electroencephalographic techniques (Giard et al., 1990; Opitz et al., 2002). PET studies (Müller et al., 2002) have revealed that these frontal sources could be in the prefrontal cortex. Similar results have also been found using fMRI (Doeller et al., 2003; Opitz et al., 2002) and intracranial recordings (Liasis et al., 2001). Electrophysiological recordings have also revealed possible frontal sources in the anterior cingulate cortex (Jemel et al., 2002; Waberski et al., 2001).

Additional sources of MMN have been located in the inferior parietal cortex (Kasai et al., 1999; Levänen et al., 1996), but its contribution is not identified in all studies. Moreover, as the relationship between structures and their temporal dynamics is still a question to be resolved, the location and nature of neural contributions to cerebral processing underlying MMN remain open questions.

In order to study the spatiotemporal dynamics of MMN cerebral sources, a two-step approach analysis has been devised. First, independent component analysis (ICA) (Makeig et al., 1996), a powerful data-driven mathematical tool that blindly separates signals' statistically independent contributions, was used to find temporally independent and spatially fixed components of ERPs. In this study, we use the Infomax ICA Algorithm (Jung et al., 2001a), in which components are obtained through minimization of mutual information among output components. ICA has recently been used to separate 
mixed information into spatially stationary and temporally independent subcomponents in some ERP studies (Jung et al., 2001b; Makeig et al., 1999, 2002) and in other branches of neuroscience and medicine (Brown et al., 2001; Calhoun et al., 2001; Nakai et al., 2004).

In the second step, the spatial maps associated with each ICA component were analyzed, with use of low-resolution tomography (LORETA) (Pascual-Marqui, 1999; Pascual-Marqui et al., 1994), to locate its cerebral sources. LORETA is a tomographic technique that gives a single solution to what is known as the inverse problem of location of cerebral sources. It is based on two constraints: it searches for the smoothest of all possible solutions, using cortical gray matter and the hippocampus of the Talairach human brain model. In the past 5 years, this tomography approach has been used in several neuroscience studies (for example, see Gomez et al., 2003; Kounios et al., 2001; Mulert et al., 2001; Pizzagalli et al., 2001). The LORETA version used in this study reconstructed the sources of activation in 2394 voxels distributed in the Talairach human brain (Pascual-Marqui, 1999).

In summary, the main aim of this study is to uncover the spatiotemporal pattern of brain activations underlying $\mathrm{MMN}$, separating statistically independent components by preprocessing data with ICA, and identifying the cerebral sources of each ICA component using LORETA.

\section{Materials and methods}

Subjects and stimuli

Sixteen healthy subjects (mean age $39 \pm 11$ years) participated in the study after having given their written consent. The paradigm described in Grau et al. (1998) was used to obtain MMN data from the subjects. The stimuli ( $85 \mathrm{~dB}$ SPL) consisted of pure sine-wave tones of $700 \mathrm{~Hz}$, with a duration of $75 \mathrm{~ms}$ (standard tone) or $25 \mathrm{~ms}$ (deviant tone), with $5 \mathrm{~ms}$ of fall/rise time. Trains of three tones were presented to subjects binaurally. The first tone of trains was standard $(P=0.5)$ or deviant $(P=0.5)$, while the other two tones were standard. The interstimulus time was $300 \mathrm{~ms}$, and the temporal separation between trains was $400 \mathrm{~ms}$. A total of 400 stimuli trains were presented randomly. Subjects were instructed to ignore auditory stimuli while they performed an irrelevant visual task (watching TV with the sound off).

\section{Recording}

EEGs (bandpass 0-100 Hz) were recorded with a SynAmps amplifier (Neuroscan Inc.) at a sampling rate of $500 \mathrm{~Hz}$. Thirty electrodes were used: 18 followed the 10-20 system without $\mathrm{O} 1$ and $\mathrm{O} 2$ (FP1, OZ, FP2, F7, F3, FZ, F4, F8, T3, C3, CZ, C4, T4, T5, P3, PZ, P4, and T6), and 12 more electrodes (FC1, FC2, FT3,

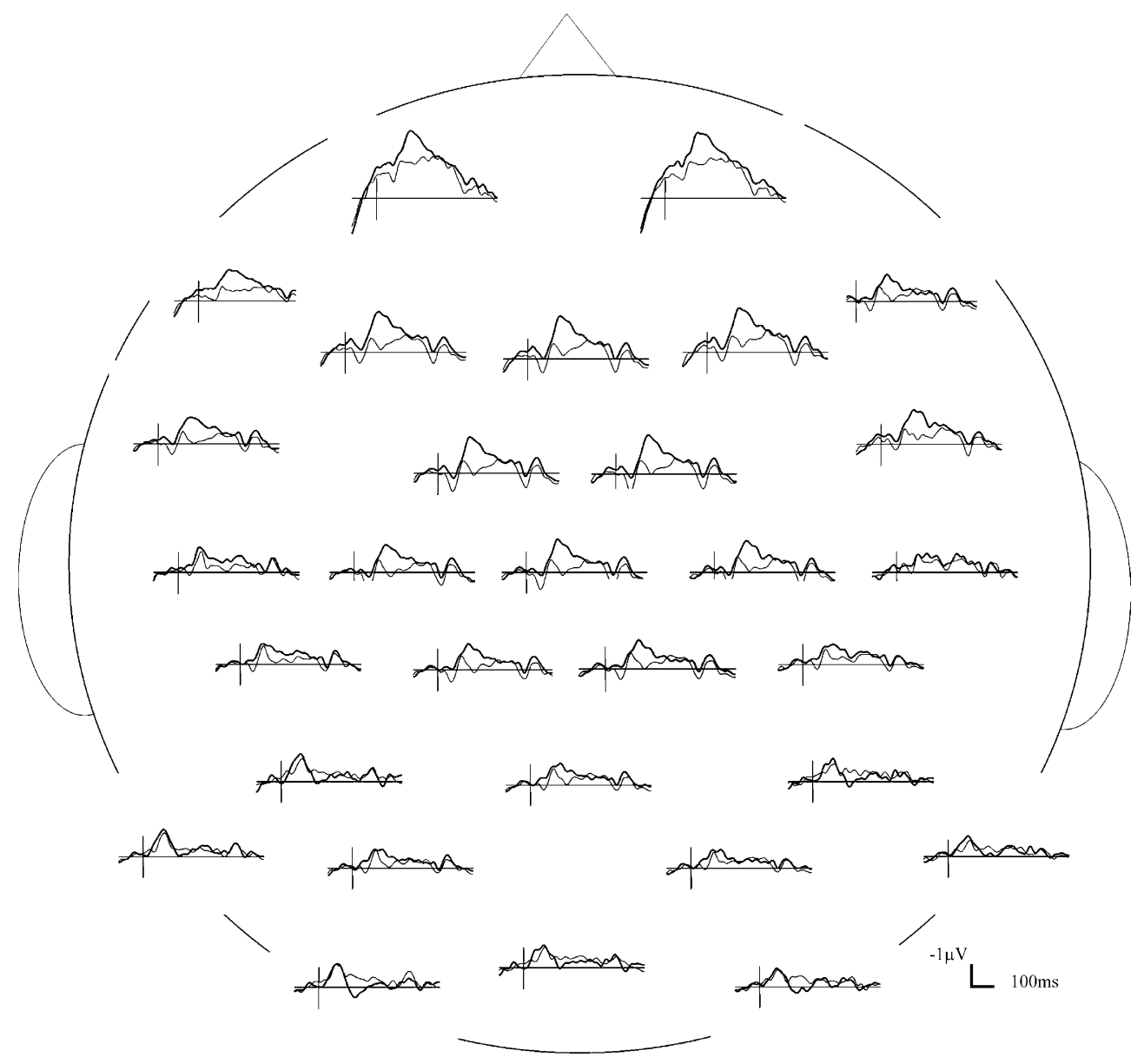

Fig. 1. Grand mean data to standard sounds (thin lines) and deviant sounds (thick line) from $100 \mathrm{~ms}$ before the onset of stimuli until $500 \mathrm{~ms}$ after. Vertical bar indicates the onset of stimuli. A clear MMN appears in frontal and central electrodes, with a polarity reversal in mastoids and adjacent electrodes. 

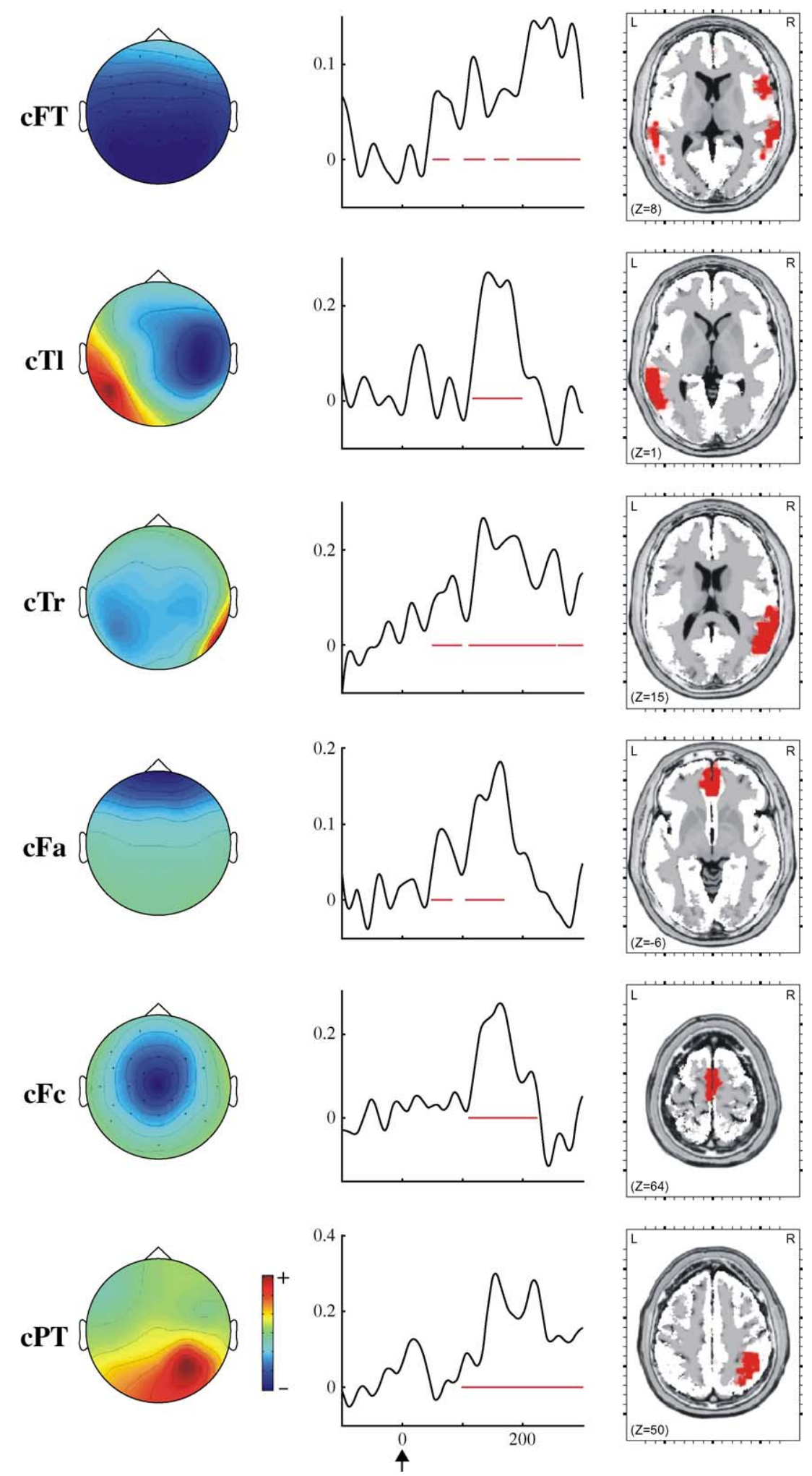

Fig. 2. Most significant independent components in the 100- to 300-ms after stimuli. The black arrow indicates the onset of stimulus. Left: maps reflecting inverse IC weights associated with each component. Middle: activations of ICs. Note that both maps of inverse IC weights and activations are unitless, and that polarity (and scaling) information is distributed across both aspects of IC. Red horizontal lines indicate activated time intervals $(P<0.05$ for $24 \mathrm{~ms})$. Right: brain sources of associated scalp maps computed by LORETA (red areas show LORETA standardized values 2.5 times greater than the standard deviation in the LORETA spatial solution). 
FT4, M1, M2, IM1, IM2, TP3, TP4, CP1, and CP2), all of them referenced to a nose electrode. Two extra electrodes were used to record vertical and horizontal ocular movements. Recordings were notch filtered at $50 \mathrm{~Hz}$. Epochs exceeding $\pm 100 \mu \mathrm{V}$ in EEG or in electrooculogram were automatically rejected. Bandpass filtering $(0.1-30 \mathrm{~Hz})$ was performed, obtaining epochs of $600 \mathrm{~ms}, 100 \mathrm{~ms}$ before stimulus to $500 \mathrm{~ms}$ after it. ERPs were found by averaging separately the first standard or deviant tone of each train (Fig. 1), and the MMN difference wave was calculated by subtracting averaged deviant ERP from averaged standard ERP for every subject. Four subjects were excluded from the rest of the study because they did not have an identifiable MMN wave.

\section{Independent component analysis tomography}

As indicated above, two-step ICA-LORETA analysis was applied to the data. For each subject, single deviant trials were subtracted from a gold standard ERP computed as the mean of the standard trials of the subject. The Infomax ICA algorithm was used in independent component analysis of concatenated MMN difference trial data from all subjects (a total of 1800 trials, 150 from each subject) (Makeig et al., 1996). Only components accounting for more than $5 \%$ of variance were selected. All the abovementioned analyses were performed using the EEGLAB (v4.3) software under Matlab@ 7.0, as described by Delorme and Makeig (2004). An independent component was considered activated if it had a sequence of at least 12 consecutive sampling points $(24 \mathrm{~ms})$ with Student's $t$ values of $P<0.05$ compared to baseline (Guthrie and Buchwald, 1991). The LORETA analysis was performed with the scalp maps associated with selected ICA components to find the generators of these maps. Only values greater than 2.5 times the standard deviation of the standardized data (in the LORETA spatial solution) were accepted as activations. This method gives the time course of each statistically independent component as well as their spatial sources. One of the main objectives of the paper was to build a bridge between classical and new ICA-based MMN approach. This is the reason for using the traditional way to study this wave (deviant minus standard ERP waves). Moreover, an alternative analysis was performed taking profit of the advantages of ICA method. An ICA was computed at both the deviant and standard data, and most activated components were selected and compared to those obtained in the aforementioned method.

\section{Results}

\section{Independent components of MMN}

Independent component analysis showed that in the MMN range (100-300 ms after the start of the stimulus) there are six main independent components that account for more than $67 \%$ of data variance. LORETA analysis of the maps associated with components revealed that two components are activated only at temporal lobe locations. We call them cTl, with sources located in the left supratemporal and middle temporal cortex, and $\mathrm{cTr}$, with sources located in the right supratemporal and middle temporal cortex. One component (cFT) had both temporal and frontal sources. The temporal sources were located in supratemporal and middle temporal cortex, in both hemispheres. The frontal source was located at the right inferior frontal cortex. Two more components $(\mathrm{cFa}$ and $\mathrm{cFc})$ had their activations in frontal sites only. $\mathrm{cFa}$ had its sources located in medial frontal cortex and anterior cingulate; whereas sources of $\mathrm{cFc}$ were also located in medial frontal cortex and cingulate, but in more central positions. Sources of the latest component (cPT) were in right inferior parietal and right supratemporal cortical separated areas (see Fig. 2).

Independent component analysis performed for each subject showed that these components were also present in single subject data (see Fig. 3). This supports the idea that ICs found with all the data are present in individual subject data. Maps related to weights of the most activated components extracted from the deviant minus standard and the ones found using ICA in both conditions were presented in Fig. 4. As can be seen, activation maps obtained with these two methods are very similar. Using the ICA-based approach, the variance explained by selected components was $79.5 \%$ for deviants and $67.96 \%$ for standards, with a higher contribution to the deviant as compared to the standard condition in all components.
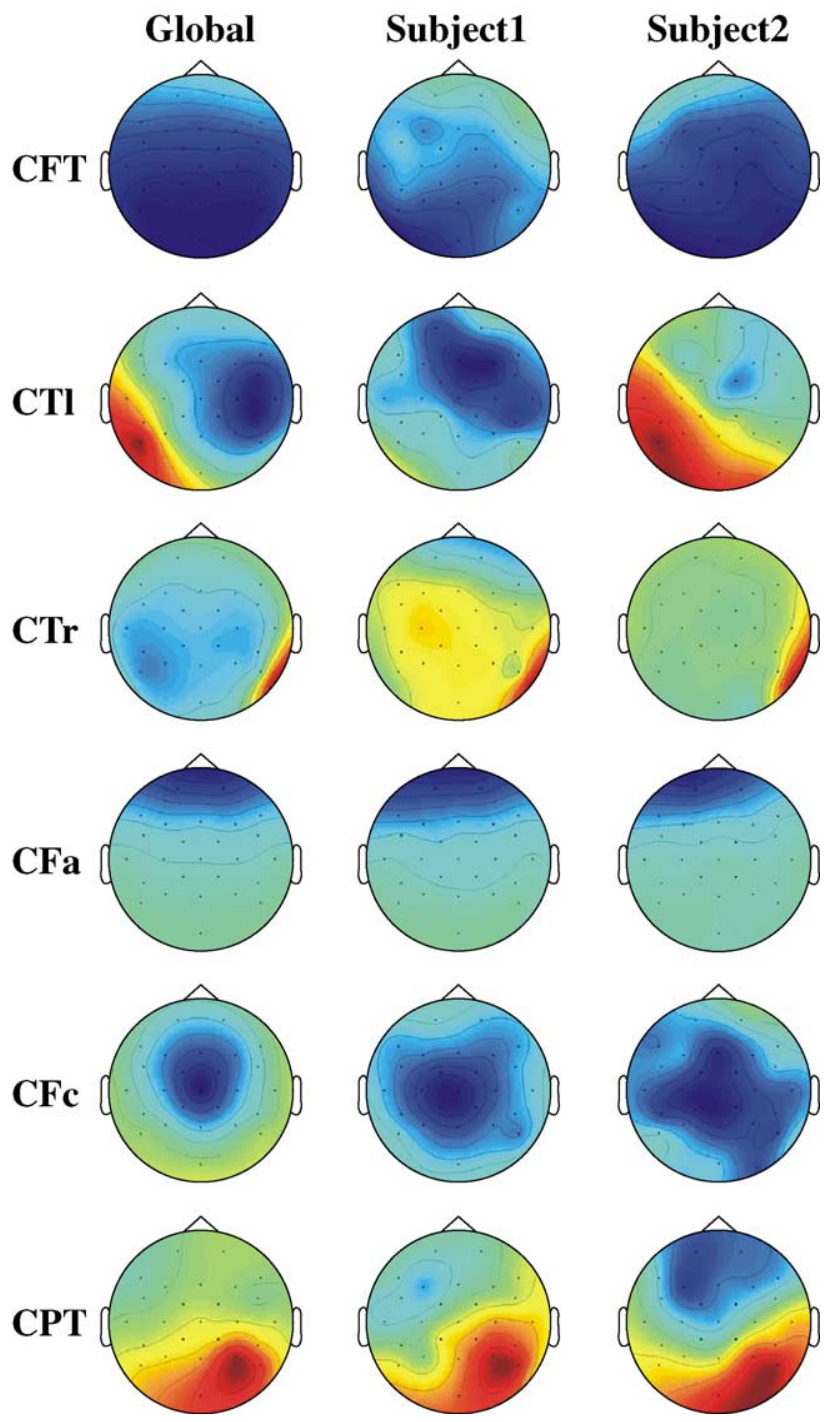

Fig. 3. Maps associated to IC weights for global data, and the corresponding maps for two selected subjects. 

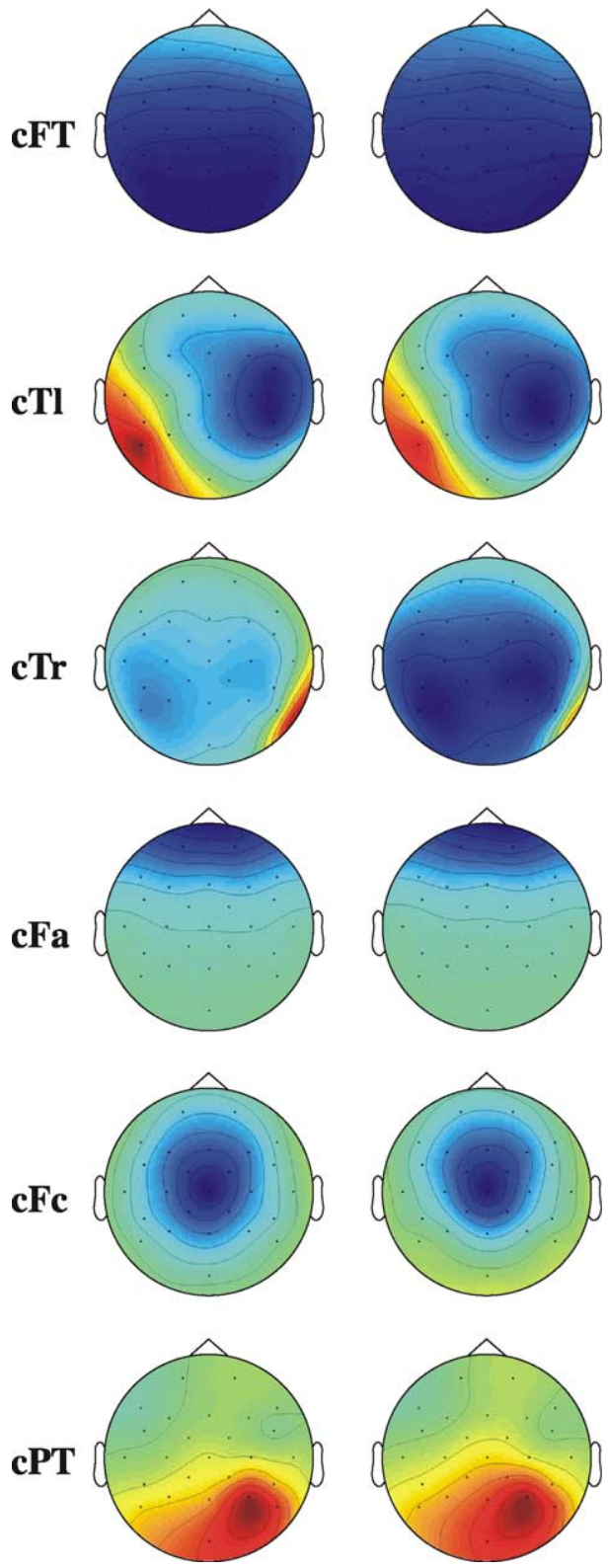

Fig. 4. Maps associated to most significant components for ICA of deviant minus standard data (left) and ICA for both deviant and standard data analyzed together (right). As can be seen, activations are very similar.

\section{Time evolution of independent components}

The time evolution of the six selected components (Fig. 2) showed that their main contribution was at 100-300 ms after stimulus onset, with some early contributions in the 50- to $100-\mathrm{ms}$ range. cFT showed an activation in the 50- to 100-ms interval, and a sequence of three more activations at 100 - to $300-\mathrm{ms}$ interval. Both cTl and cTr had activations in the 100- to 200-ms interval, but cTr remained activated until $300 \mathrm{~ms}$ after stimuli. There was an activation of $\mathrm{c} \operatorname{Tr}$ in the 50- to 100-ms interval, which was not present in cTl. cFa also had an early activation, confined to the interval 50- to 80-ms after stimulus onset. Both $\mathrm{cFa}$ and $\mathrm{cFc}$ had activations in the $100-$ to $200-\mathrm{ms}$ range, with $\mathrm{cFc}$ remaining activated until $220 \mathrm{~ms}$. Finally, cPT was activated in the entire 100to 300 -ms interval.

\section{Discussion}

To the best of our knowledge, this is the first study to combine the ICA and LORETA techniques to obtain spatially localized sources of the independent components of MMN. Six main independent components were found by ICA in the range of MMN. They were calculated using 1800 trials obtained from 12 subjects. Since they explain more than $60 \%$ of variance, their pattern could be a good signature of MMN.

All the sources associated with the six main independent components corroborate those proposed in the literature for MMN identified with different techniques of brain exploration (see Introduction), providing convergent physiological support to the validity of the present results. This corroboration includes a small contribution to right parietal cortex (see Kasai et al., 1999; Levänen et al., 1996), although the parietal activation found by Kasai appears $240 \mathrm{~ms}$ after stimuli, whereas our parietal activation covers the whole 100 - to $300-\mathrm{ms}$ interval.

Our data reflect that, in the time domain, there is orchestrated sequential incorporation and cessation of the participation of various independent components and their underlain brain structures in the processing of the auditory difference. In the 100- to 200ms interval after stimulus, all six independent components described participate. In contrast, the $200-$ to $300-\mathrm{ms}$ interval is mediated only by temporal and inferior frontal structures (i.e., cFT, CTR components) with a contribution of parietal areas (from cPT component). According to classic MMN theory (Näätänen, 1992; Rinne et al., 2000), activation differences (with respect to the standard tones) of temporal areas in the analysis of auditory difference is interpreted as a consequence of processing of changes in the physical properties of the sound; whereas activation of frontal sites is believed to reflect a process of orientation of attention to the new stimulus. So the poor frontal participation in the latter part of MMN suggests that the call to attention is confined to the first part of MMN (100-200 ms), implying frontal structures in $\mathrm{cFa}$ and $\mathrm{cFc}$, whereas processes related to analyses of auditory difference persist to $300 \mathrm{~ms}$, including a residual frontal contribution from $\mathrm{cFT}$.

According to our results, sources of independent components can be generated by one or more spatially separated sources. Although most components have a compact source (such as cTl, $\mathrm{cTr}, \mathrm{cFa}$, and $\mathrm{cFc}$ ), others have two (cPT) or even three (cFT) separated sources. This partially confirms results found by Makeig et al., in which each independent component was forced to associate with a single dipole generator (Makeig et al., 1999, 2002) or two of them (Makeig et al., 2004). However, our tomographic method has the advantage of not making a priori assumptions on the number of sources, which opens the possibility of finding multiple sources underlying a single component.

In our study, the right hemisphere dominance repeatedly described in MMN (Näätänen, 1992) is supported by (1) the participation of $\mathrm{cTr}$ in the whole MMN range, whereas $\mathrm{cTl}$ is only present in the earlier part of MMN; (2) the participation of only the right hemisphere in $\mathrm{CPT}$; and (3) the right frontal contribution of cFT.

Several functional points can be drawn from these findings. A specific operation of brain computing (analysis of auditory difference) is underlain by several statistically independent processes captured by different independent components and supported by identifiable distant brain sources. Moreover, our results support the view that a specific brain structure can participate in different components, working simultaneously in 
more than one brain computing process. This is the case of the left temporal cortex, present in $\mathrm{cTl}$ and $\mathrm{cFT}$, and of the right temporal cortex, participating in $\mathrm{cFT}$, cTr, and $\mathrm{cPT}$. The spatial overlapping of sources of independent components would be explained by the limited resolution of tomographic method used that could not fully separate neighboring neural populations participating in distinct processing tasks, or by the contribution of intermixed neuronal populations located in the same brain structure performing different brain computation processes.

The main limitation of the present study is the use of only 30 electrodes. Although the results found with the ICA-LORETA proposed method seem reasonable, it would be interesting to replicate and refine the same results with the use of a greater number of electrodes that could modify the number of independent components found. Future studies also need to be carried out to determine the functional role of each component of $\mathrm{MMN}$, to reveal if they correspond to physiologically distinct/decoupled processes, and to clarify whether observed patterns are similar in other cerebral processing mechanisms. One field of application of present results in MMN would be the study of changes in the spatiotemporal behavior of independent components and their associated sources in various brain-related pathologies.

In conclusion, this work proposes the study of MMN with a two-step analysis approach, based on the combined capacities of ICA and LORETA, to reveal the spatiotemporal dynamics of its cerebral sources. ICA explains what (independent) processes are involved in the brain computation of the difference between two tones, whereas LORETA models the possible sources of these processes. The synergy between ICA and LORETA gives evidence that the cerebral processing underlying auditory change detection consists of six main statistically independent components, whose sources (frontal, temporal, and parietal) are clearly identified in time and space.

\section{Acknowledgments}

The authors would like to thank María Dolores Polo and Carles Escera for their help in data collection. We are also grateful to Arnaud Delorme, Scott Makeig, and Roberto Pascual-Marqui for their generous gift of software. This research was supported by grants from the Generalitat de Catalunya (Xarxa Temàtica "Psicofisiologia Cognitiva i Neurodinàmica Clínica"), the Ministerio de Ciencia y Tecnologia (BSO2000-0679), and the European Union (FP6-507231, Sensation) to Carles Grau, and by the internal Kolmogorov Project and the EU FP6 Sensation Project at Starlab.

\section{References}

Alain, C., Woods, D.L., Knight, R.T., 1998. A distributed cortical network for auditory sensory memory in humans. Brain Res. 812, 23-37.

Brown, G.D., Yamada, S., Sejnowski, T.J., 2001. Independent component analysis at the neural cocktail party. Trends Neurosci. 24, 54-63.

Calhoun, V.D., Adali, T., Pearlson, G.D., Pekar, J.J., 2001. Spatial and temporal independent component analysis of functional MRI data containing a pair of task-related waveforms. Hum. Brain Mapp. 13, $43-53$.

Delorme, A., Makeig, S., 2004. EEGLAB: an open source toolbox for analysis of single-trial EEG dynamics. J. Neurosci. Methods 134, 9-21.

Doeller, C.F., Opitz, B., Mecklinger, A., Krick, C., Reith, W., Schröger, E., 2003. Prefrontal cortex involvement in preattentive auditory deviance detection: neuroimaging and electrophysiological evidence. NeuroImage 20, 1270-1282.

Giard, M.H., Perrin, F., Perrier, J., Bouchet, P., 1990. Brain generators implicated in the processing of auditory stimulus deviance: a topographic event-related potential study. Psychophysiology 27, 627-640.

Gomez, C., Marco, J., Grau, C., 2003. Preparatory visuo-motor cortical network of the contingent negativity variation estimated by current density. NeuroImage 20, 216-224.

Grau, C., Escera, E., Yago, E., Polo, M.D., 1998. Mismatch negativity and auditory sensory memory evaluation: a new faster paradigm. NeuroReport 9, 2451-2456.

Guthrie, D., Buchwald, J.S., 1991. Significance testing of difference potentials. Psychophysiology 28, 240-244.

Jääskeläinen, I.P., Ahveninen, J., Bonmassar, G., Dale, A.M., Ilmoniemi, R.J, Levänen, S., Lin, F., May, P., Melcher, J., Stufflebeam, S., Tiitinen, H., Belliveau, J.W., 2004. Human posterior auditory cortex gates novel sounds to consciousness. Proc. Natl. Acad. Sci. 101, 6809-6814.

Jemel, B., Achenbach, C., Müller, B.W., Röpcke, B., Oades, R.D., 2002. Mismatch negativity results from bilateral asymmetric dipole sources in the frontal and temporal lobes. Brain Topogr. 15, 13-27.

Jung, T.P., Makeig, S., Mckeown, M.J., Bell, A.J., Lee, T.W., Sejnowski, T.J., 2001a. Imaging brain dynamics using independent component analysis. Proc. IEEE 89, 1107-1122.

Jung, T.P., Makeig, S., Westerfield, M., Townsend, J., Courchesne, E., Sejnowski, T.J., 2001b. Analysis and visualization of single-trial eventrelated potentials. Hum. Brain Mapp. 14, 166-185.

Kasai, K., Nakagome, K., Itoh, K., Koshida, I., Hata, A., Iwanami, A., Fukuda, M., Hiramatsu, K.I., Kato, N., 1999. Multiple generators in the auditory discrimination process in humans. NeuroReport 10 , 2267-2271.

Kircher, T.J., Rapp, A., Grodd, W., Buchkremer, G., Weiskopf, N., Lutzenberger, W., Ackermann, H., Mathiak, K., 2004. Mismatch negativity responses in schizophrenia: a combined fMRI and wholehead MEG study. Am. J. Psychiatry 161, 294-304.

Kounios, J., Smith, R.W., Yang, W., Bachman, P., D’Esposito, M., 2001. Cognitive association formation in human memory revealed by spatiotemporal brain imaging. Neuron 29, 297-306.

Levänen, S., Ahonen, A., Hari, R., McEvoy, L., Sams, M., 1996. Deviant auditory stimuli activate human left and right auditory cortex differently. Cereb. Cortex 2, 288-296.

Liasis, A., Towell, A., Alho, K., Boyd, S., 2001. Intracranial identification of an electric frontal-cortex response to auditory stimulus change: a case study. Cognit. Brain Res. 11, 227-233.

Makeig, S., Bell, A.J., Jung, T.P., Sejnowski, B., 1996. Independent component analysis of electroencephalographic data. Adv. Neural Inf. Process Syst. 8, 145-151.

Makeig, S., Westerfield, M., Jung, T.P., Covington, J., Townsend, J., Sejnowski, B., Couchesne, E., 1999. Functionally independent components of the late positive event-related potential during visual spatial attention. J. Neurosci. 19, 2665-2680.

Makeig, S., Westerfield, M., Jung, T.P., Enghoff, S., Townsend, J., Couchesne, E., Sejnowski, B., 2002. Dynamic brain sources of visual evoked responses. Science 295, 690-694.

Makeig, S., Debener, S., Onton, J., Delorme, A., 2004. Mining eventrelated brain dynamics. Trends Cogn. Sci. 134, 9-21.

Mulert, C., Gallinat, J., Pascual-Marqui, R.D., Dorn, H., Frick, K., Schlattmann, P., Mientus, S., Herrmann, W.M., Winterer, G., 2001. Reduced event-related current density in the anterior cingulate cortex in schizophrenia. NeuroImage 13, 589-600.

Müller, B.W., Jüpter, M., Jentzen, W., Müllert, P., 2002. Cortical activation to auditory mismatch elicited by frequency deviant and complex novel sounds: a PET study. NeuroImage 17, 231-239.

Nätänen, R., 1992. Attention and Brain Function. Erlbaum.

Näätänen, R., Gaillard, A.W.K., Mantysalo, S., 1978. Early selectiveattention effect on evoked potential reinterpreted. Acta Psychol. 42, $313-329$.

Näätänen, R., Tervaniemi, M., Sussmann, E., Paavilainen, P., Winkler, I., 
2001. 'Primitive intelligence' in the auditory cortex. Trends Neurosci. $24,283-288$.

Nakai, T., Muraki, S., Bagarinao, E., Miki, Y., Takehara, Y., Matsuo, Y., Kato, C., Sakahara, H., Isoda, H., 2004. Application of independent component analysis to magnetic resonance imaging for enhancing the contrast of gray and white matter. NeuroImage 21, 251-260.

Opitz, B., Rinne, T., Mecklinger, A., Cramon, D.Y., Schröger, E., 2002. Differential contribution of frontal and temporal cortices to auditory change detection: fMRI and ERP results. NeuroImage 15, 167-174.

Pascual-Marqui, R.D., 1999. Review of methods solving the EEG inverse problem. Int. J. Bioelectromagn. 1, 75-86.

Pascual-Marqui, R.D., Michel, C.M., Lehmann, D., 1994. Low resolution electromagnetic tomography: a new method for localizing electrical activity in the brain. Int. J. Psychophysiol. 18, 49-65.

Pizzagalli, D., Pascual-Marqui, R.D., Nitschke, J.B., Oakes, T.R., Larson, C.L., Abercrombie, H.C., Schaefer, S.M., Koger, J.V., Benca, R.M.,
Davidson, R.J., 2001. Anterior cingulate activity as a predictor of degree of treatment response in major depression: evidence from brain electrical tomography analysis. Am. J. Psychiatry 158, $405-415$

Rinne, T., Alho, K., Ilmoniemi, J., Virtanen, J., Näätänen, R., 2000. Separate time behaviors of the temporal and frontal mismatch negativity sources. NeuroImage 12, 14-19.

Rosburg, T., Haueisen, J., Kreitschmann-Andermahr, I., 2004. The dipole location shift within the auditory evoked neuromagnetic field components $\mathrm{N} 100 \mathrm{~m}$ and mismatch negativity (MMNm). Clin. Neurophysiol. $115,906-913$.

Waberski, T.D., Kreischmann-Andermahr, I., Kawohl, W., Darvas, F., Ryang, Y., Gobbele, R., Buchner, H., 2001. Spatio-temporal source reveals subcomponents of the human auditory mismatch negativity in the cingulate and right inferior temporal gyrus. Neurosci. Lett. 308, 107-110. 